\title{
Applicability of Near-Infrared Spectroscopy to Measure Cerebral Autoregulation Noninvasively in Neonates: A Validation Study in Piglets
}

\author{
GITTE H. HAHN, CHRISTIAN HEIRING, OLE PRYDS, AND GORM GREISEN
}

\begin{abstract}
Department of Neonatology [G.H.H., G.G.], Copenhagen University Hospital-Rigshospitalet, DK-2100 Copenhagen, Denmark; Department of Pediatrics [C.H.], Hillerød Hospital, DK-3400 Hillerød, Denmark; Department of Pediatrics [O.P.], Copenhagen University

Hospital-Hvidovre Hospital, DK-2650 Hvidovre, Denmark
\end{abstract}

\begin{abstract}
Impaired cerebral autoregulation (CA) is common and is associated with brain damage in sick neonates. Frequency analysis using spontaneous changes in arterial blood pressure (ABP) and cerebral near-infrared spectroscopy (NIRS) has been used to measure CA in several clinical studies. Coherence of the NIRS and ABP signals (i.e. correlation in the frequency domain) detects impairment of $\mathrm{CA}$, whereas gain (i.e. magnitude of $\mathrm{ABP}$ variability passing from systemic to cerebral circulation) estimates the degree of this impairment. So far, however, this method has not been validated. In 12 newborn piglets, we compared NIRS-derived measures of CA with a conventional measure of CA: cerebral blood flow was measured by laser Doppler flowmetry, and changes in ABP were induced by inflating a thoracic aorta balloon. CA capacity was calculated as $\% \Delta \mathrm{CVR} / \% \Delta \mathrm{ABP}$ (i.e. percentage of full autoregulatory capacity), where CVR (i.e. cerebral vascular resistance) was estimated as ABP/Doppler flux. Correlation between coherence and CA capacity ( $r=-0.34, n=24, p>0.05)$ and between gain and CA capacity ( $r=-0.11, n=24, p>0.05)$ was limited. As expected, however, gain was significantly associated with CA capacity in measurements with significant coherence $(r=-0.55, n=15, p=0.03)$. In conclusion, our data validate frequency analysis for estimation of CA in clinical research. Low precision, however, hampers its clinical application. (Pediatr Res 70: 166-170, 2011)
\end{abstract}

$\mathrm{C}$ erebral autoregulation (CA) is a physiological mechanism that cushions the cerebral circulation from transient changes in arterial blood pressure (ABP). With intact CA, cerebral blood vessels dilate proportional to decreases in $\mathrm{ABP}$ and constrict with increases in ABP. In that way, cerebral blood flow $(\mathrm{CBF})$ remains approximately constant over a wide range of $\mathrm{ABP}$ (1). CA may be disrupted not only in sick preterm infants but also in those that are clinically well (2). However, there is evidence that $\mathrm{CBF}$ is independent from $\mathrm{ABP}$ over a wide pressure range in preterm infants, suggesting that CA may actually be effective in the immature brain (3). In preterm infants, disrupted CA has been associated with development of intraventricular hemorrhage (IVH) and periventricular leukomalacia $(4,5)$.

Received September 15, 2010; accepted February 10, 2011.

Correspondence: Gitte H. Hahn, M.D., Department of Neonatology, 5021 Rigshospitalet, Blegdamsvej 9, DK-2100 København Ø, Denmark; e-mail: gwhahn@dadlnet.dk

Supported by the Ludvig and Sara Elsass Foundation and the University of Copenhagen, Denmark.
Impairment of CA narrows the range of $\mathrm{ABP}$ where $\mathrm{CBF}$ is matched to metabolic needs causing a state with vulnerability to even modest changes in ABP. Therefore, optimal management of $\mathrm{ABP}$ would include a possibility to titrate $\mathrm{ABP}$ into that range, where $\mathrm{CA}$ is working most effectively. However, to date, no monitoring device that measures CA continuously exists. Near-infrared spectroscopy (NIRS) detects changes in cerebral oxygenation noninvasively and continuously. Assuming that these changes represent changes in $\mathrm{CBF}$, frequency analysis yielding coherence and gain between spontaneous changes in ABP and cerebral NIRS could fill that role. Coherence (i.e. correlation in the frequency domain) is supposed to detect impairment of $\mathrm{CA}$, whereas gain estimates the magnitude of this impairment (i.e. amount of $\mathrm{ABP}$ variability passing from systemic to cerebral circulation). A low gain would indicate that although CA was not perfect, at least the magnitude of changes in cerebral oxygenation was small or moderate. Similarly, a high gain would indicate that even moderate changes in ABP were associated with large changes in cerebral oxygenation.

Physiological adjustments of CBF occur both quickly and slowly $(6,7)$. This temporal heterogeneity of CA is accounted for when correlation between cerebral NIRS and changes in $\mathrm{ABP}$ is analyzed in the frequency domain. Furthermore, analysis in the frequency domain eliminates the effect of time lag between changes in ABP and cerebral NIRS as a consequence of cerebrovascular transit time being $\sim 10 \mathrm{~s}$ in neonates (8).

Frequency analysis between spontaneous changes in ABP and cerebral NIRS has been used in several clinical studies of preterm infants to measure $\mathrm{CA}$ and has demonstrated that impaired CA is frequent (9) and associated with IVH $(10,11)$ and mortality (12) in this vulnerable group of patients. However, to our knowledge, so far no studies have validated reliability of this method against a conventional measurement of CA. Within the time domain, Brady et al. (13) studied the correlation between cerebral NIRS and spontaneous ABP

\footnotetext{
Abbreviations: ABP, arterial blood pressure; CA, cerebral autoregulation; $\mathbf{C B F}$, cerebral blood flow; CVR, cerebrovascular resistance; $\mathbf{C V R}_{\mathbf{e}}$, estimated cerebrovascular resistance; IVH, intraventricular hemorrhage; LDF, laser Doppler flowmetry; LF, low frequency $(0.003-0.04 \mathrm{~Hz})$; MAP, mean arterial blood pressure; NIRS, near-infrared spectroscopy; OI, oxygenation index; PSD, power spectral density; VLF, very low frequency $(0.04-0.1 \mathrm{~Hz})$
} 
changes in a piglet model of slowly progressing hypotension and found it to be sensitive for loss of CA when compared with laser Doppler flowmetry (LDF).

We aimed to validate the use of frequency analysis to measure coherence and gain between cerebral NIRS and spontaneous fluctuations in ABP for noninvasive measurement of $\mathrm{CA}$ by comparing it with a conventional measurement of $\mathrm{CA}$ in piglets using induced changes in $\mathrm{ABP}$ and $\mathrm{LDF}$.

\section{METHODS}

The study was approved by the National Committee on Animal Experimentation, Denmark.

Anesthesia. In 12 piglets, aged 1-3 d (median age, $1.5 \mathrm{~d}$ ) and weighing $1.4-2.0 \mathrm{~kg}$ (median weight, $1.6 \mathrm{~kg}$ ), anesthesia was induced by inhalation of $3 \%$ isoflurane followed by an i.v. induction dose of propofol $(5 \mathrm{mg} / \mathrm{kg})$. Analgesia for the surgical procedures was secured with an i.v. bolus of fentanyl $(20 \mu \mathrm{g} / \mathrm{kg})$. A tracheostomy was performed, and mechanical ventilation was instituted. Isoflurane inhalation was stopped, and anesthesia was maintained with i.v. propofol $(15-25 \mathrm{mg} / \mathrm{kg} / \mathrm{h})$ for the duration of the experiment. We choose this anesthetic regime to avoid the known effect of inhaled anesthetics on cerebrovascular responsiveness during the experiment (14). Fraction of inspired oxygen and ventilation were adjusted to keep arterial saturation at 95 to $98 \%$ and partial pressure of $\mathrm{CO}_{2}$ in arterial blood at 4 to $6 \mathrm{kPa}$.

Surgical preparation and monitoring of animals. The femoral arteries were cannulated bilaterally for placement of the following: 1) a pressure and blood gas monitoring line and 2) a 4-Fr catheter with an inflatable balloon near its tip (Fogarty Fortis, Arterial Embolectomy Catheter, Edwards Lifesciences). Inflation of this balloon occluded the lumen of the thoracic part of aorta resulting in gradual cerebral hypertension. Position of the Fogarthy catheter was checked with inflation of the balloon: first inflation with the tip of the pressure line placed below the tip of the Fogarthy catheter causing a flat arterial pressure curve. Then the pressure line was further advanced until inflation of the balloon caused hypertension with an intact arterial pressure curve. The femoral vein was cannulated for placement of a central venous line for drug and fluid infusion (4 Fr double lumen catheter). Infusion of glucose $(10 \%, 2.5 \mathrm{~mL} / \mathrm{kg} / \mathrm{h})$ was maintained throughout the experiment. Finally, all incisions were infiltrated with bupivacaine $(2.5 \mathrm{mg} / \mathrm{mL})$. All piglets received ampicillin $(200 \mathrm{mg} / \mathrm{kg})$ and gentamicin $(4 \mathrm{mg} / \mathrm{kg})$ as a single dose before catheterization.

Piglets were kept on a warming pad to maintain rectal temperature at $38.5^{\circ} \mathrm{C}$ to $39.5^{\circ} \mathrm{C}$. Arterial saturation and heart rate was monitored by pulse oximetry from the forepaw (Radical 7, Masimo, Hannover, Germany).

Near-infrared spectroscopy. NIRS data were recorded using a NIRO-300 spectrophotometer (Hamamatsu Photonics, Hamamatsu City, Japan). The probes were fixed in a nontransparent, soft probe holder with an interoptode distance of $4 \mathrm{~cm}$ and placed above the eye, across the frontal and parietal cortex. A self-adhesive band (Coban, $3 \mathrm{M}$ ) was used to secure its position over time (Fig. 1). Changes in the relative concentrations of oxygenated and deoxygenated $\mathrm{Hb}$ were recorded and used to calculate the oxygenation index (OI). OI is the difference between oxygenated and deoxygenated Hb divided by a factor of 2 . Changes in OI have been validated to represent changes in CBF in several animal studies (15-17).

Laser Doppler. A craniotomy (diameter: $4 \mathrm{~mm}$ ) was performed for parietal placement of a LDF probe without perforating the dura (Perimed 5010, Stockholm, Sweden), opposite the side of the NIRS probes. To secure stable measurements from the same area of cerebral cortex over time, the probe was placed in a specially designed metal washer glued to the skull, and positioned to avoid high baseline flux values secondary to placement over large vessels (Fig. 1). Correct position of the probe was checked by inflation of the thoracic aorta balloon after inhalation of $3 \%$ isoflurane for $1 \frac{1}{2} \mathrm{~min}$, known to abolish CA (14). Position of the probe was only accepted if Doppler flux increased in parallel with ABP, thereby eliminating noise to cause the observed changes in Doppler flux during balloon inflations. We checked the position before beginning of the experiment and again before each series of measurement during baseline measurements, where changes in Doppler flux were minimal.

Signal sampling. ABP waveforms were measured with a pressure transducer connected to the arterial catheter. Before the mean ABP (MAP) was calculated, the ABP data were filtered by a first-order low-pass filter with a cutoff frequency of $0.3 \mathrm{~Hz}$. NIRS, LDF, and MAP data were sampled simultaneously and synchronized with arterial saturation at $2 \mathrm{~Hz}$ by an $\mathrm{AD}$ converter and stored on a laptop for further analysis. All signals were displayed throughout the study (LabVIEW, National Instruments).

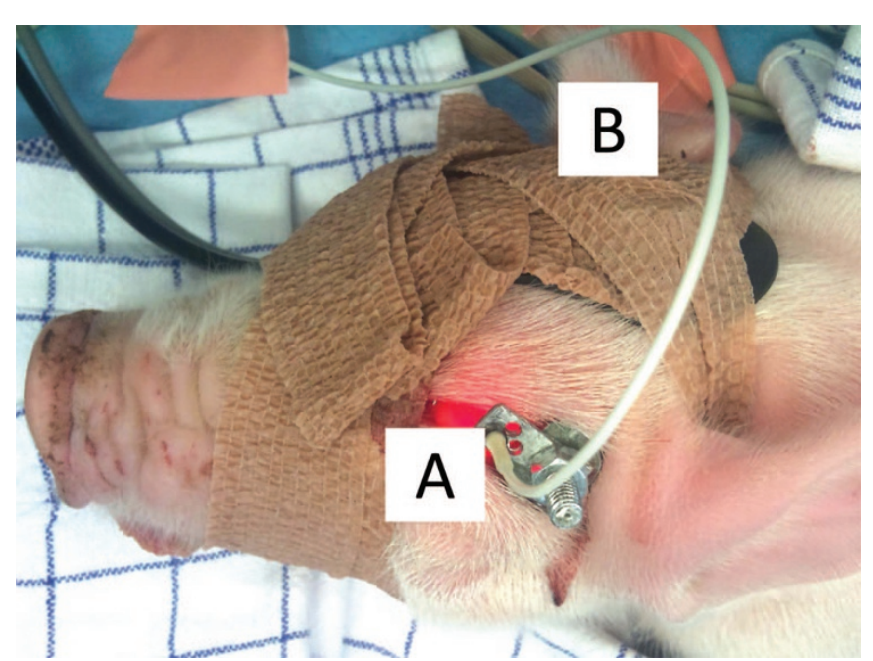

Figure 1. Picture showing the placement of the LDF probe $(A)$ and NIRS optodes $(B)$ on the head of a piglet. The LDF probe was placed in a specially designed metal washer that was glued to the skull enabling stable measurements from the same area of cerebral cortex over time. The tip of the probe was placed in a 4-mm craniotomy in contact with dura. The NIRS-optodes were fixed in a nontransparent, soft probe holder and placed above the eye, across the frontal and parietal cortex opposite the site of the craniotomy. We used a self-adhesive bandage to secure its position over time.

Experimental protocol. Piglets were studied during baseline and during hypovolemia (induced by withdrawal of $1 / 3$ of the estimated blood volume $(\mathrm{mL})$ (weight $(\mathrm{g}) \times 0.07 \times 1 / 3)$. Hypovolemia was induced to validate the method over a range of hemodynamic conditions. In both states, spontaneous changes in ABP and cerebral oxygenation (NIRS) were monitored for $40 \mathrm{~min}$. Before and after this period of spontaneous changes in ABP, the thoracic aorta balloon was inflated six times (three times with half its maximum volume and subsequently three times with its maximum volume). Inflations lasted $30 \mathrm{~s}$ and pauses lasting $30 \mathrm{~s}$ were interpolated (corresponding to a frequency of 0.02 $\mathrm{Hz}$ ). CA capacity was calculated from changes in cortical flux (LDF probe) (Fig. 2). After each experiment, the piglet was killed under general anesthesia with i.v. pentobarbitone $(100 \mathrm{mg} / \mathrm{kg})$.

Calculation of NIRS derived measures of CA (coherence and gain). Transfer function analysis of coherence and gain between continuous measurements of spontaneous changes in OI and MAP were computed in two frequency bands: 1) the very low frequency range (VLF; 0.003-0.04 Hz) and the low frequency range (LF; $0.04-0.1 \mathrm{~Hz}$ ), corresponding to periodic variations occurring over 25-300 and 10-25 s (Matlab, Math Works). The numeric value of coherence ranges between 0 and 1 , with 1 indicating perfect frequency-specific correlation (i.e. lack of CA) and 0 indicating complete lack of correlation (i.e. perfectly working CA) (7). Data were divided into 10-min epochs, which were subdivided into three segments of $5 \mathrm{~min}$ with $50 \%$ overlap, and a Hanning-window was applied to minimize spectral leakage. Time signals of OI and MAP were then detrended and transformed into power

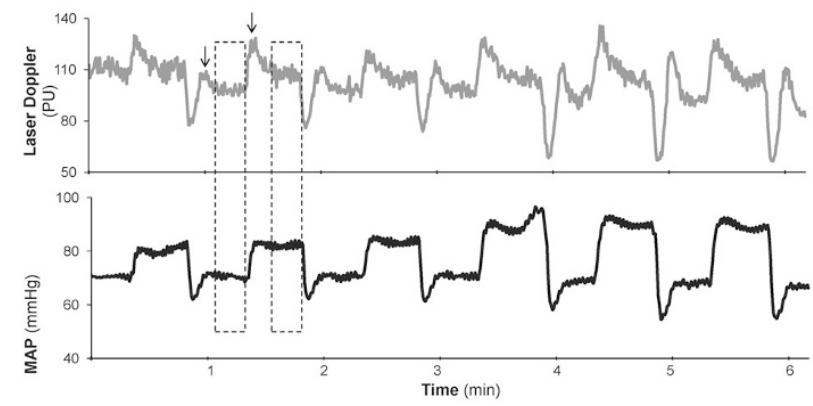

Figure 2. Changes in cortical laser Doppler flux (PU, i.e arbitrary units; gray lines) to induced rises in $\mathrm{ABP}$ ( $\mathrm{mm} \mathrm{Hg}$; black lines). The boxes illustrate the last $15 \mathrm{~s}$ of baseline (aortic balloon not inflated) and rise (aortic balloon inflated) in ABP used to calculate CA capacity. Note the transient increase in cortical laser Doppler flux as response to changes in ABP (arrows). The last three aortic balloon inflations were made with maximum volume. 
spectral density (PSD) by Fourier transform. PSD is the "power" of signals in the frequency domain and measures their variance at specific frequencies. For each 10-min epoch, coherence and gain were averaged over the frequency band. Moreover, to reduce possible measurement noise caused by ABP sometimes vary much and sometimes little, we weighted these repeated measurements of coherence and gain with amount of variability in ABP in form of transfer power, i.e. epochs with high variation in $\mathrm{ABP}$ were weighted in favor of those with low variation in ABP. This procedure left us with a single weighted mean of coherence and gain in each piglet during baseline and hypovolemia, respectively. As described in details in an earlier work, we used Monte Carlo simulations to find the level of significant coherence ( $\geq 0.47$ in VLF and $\geq 0.45$ in LF) (18). In short, OI and MAP signals were reshuffled randomly 10,000 times. These 10,000 values were then sorted in ascending order, and the $95 \%$ percentile was taken as the $95 \%$ CI of significant coherence. In physiological terms, statistically significant coherence implies that $\mathrm{CBF}$, to some extent, varies with changes in $\mathrm{ABP}$.

Low spontaneous variability in ABP decreases the signal-to-noise ratio, and consequently physiological and instrumental noise dominates the cerebral oxygenation. This can incorrectly deem a measurement as having insignificant coherence. To estimate the impact of noise, we tested whether PSD of MAP and estimated instrumental or physiological noise differed between measurements with significant and insignificant coherence. We estimated instrumental noise as the spontaneous $1 / 2$-sec variation of OI during $10 \mathrm{~min}$ of measurements. Physiological noise was then estimated as follows: $\sqrt{(1-\text { coherence }) \times P S D(O I)}-$ noise $_{1}$, where noise I $_{\mathrm{I}}$ is the estimated instrumental noise.

Calculation of laser Doppler derived measures of CA (CA capacity). CA capacity was calculated as described by Tiecks et al. (19). We calculated percentage change in estimated cerebrovascular resistance $\left(\mathrm{CVR}_{\mathrm{e}}\right)$ in relation to change in ABP. To allow steady state to be obtained, we used a mean over the last $15 \mathrm{~s}$ during baseline (1) and during each balloon-induced ABP rise (2) (Fig. 2). We estimated $\mathrm{CVR}$ as $\mathrm{CVR}_{\mathrm{e}}=\mathrm{ABP} /$ laser Doppler flux and calculated static CA capacity as CA capacity $=\left(\% \Delta \mathrm{CVR}_{\mathrm{e}} \mathrm{e} \% \Delta \mathrm{ABP}\right) \times 100 \%$ with $\% \Delta \mathrm{CVR}_{\mathrm{e}}=\left(\mathrm{CVR}_{\mathrm{e}} 2-\mathrm{CVR}_{\mathrm{e}} 1\right) / \mathrm{CVR}_{\mathrm{e}} 1$ and $\% \Delta \mathrm{ABP}=(\mathrm{ABP} 2-$ $\mathrm{ABP} 1) / \mathrm{ABP}$. Thus, CA capacity is expressed as a percentage of full autoregulatory capacity. Hence, a rise in $\mathrm{ABP}$ that is fully compensated by a rise in CVR, i.e. perfectly working CA, would yield a CA capacity of $100 \%$. Similarly, a CA capacity of $0 \%$ arises if CVR does not change at all, i.e. totally impaired CA. Finally, we calculated a mean value of CA capacity in each piglet during both baseline and hypovolemia.

Statistics. In general, we used parametric methods when normality could be obtained and nonparametric methods if this was not the case. Pearson correlation coefficient $r$ was used to measure the strength of the correlation between all paired averages for CA capacity and coherence and gain in both frequency ranges (SPSS statistics 17.0). Before these analyses, normality was checked visually and with Shapiro-Wilk $W$ test for normality. To obtain normality, gain was transformed reciprocally (i.e. gain ${ }^{-1}$ ). We used the Mann-Whitney $U$ test to test whether noise and PSD of MAP differed between measurements with significant and insignificant coherence. Wilcoxon-signed rank test was used to study how PSD of MAP differed between VLF and LF. A two-tailed $p$ value of 0.05 was considered significant.

\section{RESULTS}

NIRS derived measures of CA (coherence and gain). Fifteen (63\%) of the 24 measurements (two in each piglet) had significant coherence in VLF. In LF, the same number was 10 (42\%). Amount of spontaneous variation in MAP (i.e. PSD of MAP), instrumental noise, and physiological noise did not differ significantly between measurements with and without significant coherence (data not shown). Median amount of spontaneous variation in MAP was significantly higher in VLF compared with LF (10.7 versus $1.4 \mathrm{~mm} \mathrm{Hg}^{2}$, $p<0.001$ ). The overall mean ( \pm SEM) coherence in all piglets was 0.54 $( \pm 0.03)$ in VLF and $0.45( \pm 0.02)$ in LF. Overall median gain was $0.11(0.05-0.51) \mu \mathrm{M} / \mathrm{mm} \mathrm{Hg}$ in VLF and $0.11(0.06-$ $0.82) \mu \mathrm{M} / \mathrm{mm} \mathrm{Hg}$ in $\mathrm{LF}$.

Laser-Doppler-derived measures of CA (CA capacity). A total of 258 induced ABP rises in the 12 piglets were obtained in which ABP and cortical laser Doppler flux reached steady state (135 during baseline and 123 during hypovolemia). In each piglet repeated measurements of CA capacity were averaged in both states, yielding a total of 24 measurements. The overall mean CA capacity in all piglets was $54 \%( \pm 5.4 \%)$.

Correlation between NIRS and laser Doppler-derived measures of $\boldsymbol{C A}$. In VLF correlation between coherence and CA capacity $(r=-0.34, n=24, p>0.05)$ and between gain and CA capacity ( $r=-0.11, n=24, p>0.05$ ) was poor. However, for the subgroup of measurements with significant coherence (i.e. coherence exceeding the limit of 0.47), there was a strong, significant correlation between gain and CA capacity $(r=-0.55, n=15, p=0.03)$ with high gain associated with low CA capacity (Fig. 3).

In LF, all correlations were poor and insignificant $(r=0.01$ for coherence, $r=0.13$ for gain and $r=-0.004$ for gain in the subgroup with significant coherence).

Two illustrative examples of $40 \mathrm{~min}$ measurements of cerebral NIRS (OI) obtained during spontaneous changes in $\mathrm{ABP}$ are shown in Fig. 4. In both measurements, coherence was significant (VLF range). However, gain, i.e. the amount of spontaneous variation in $\mathrm{ABP}$ which was being transferred to
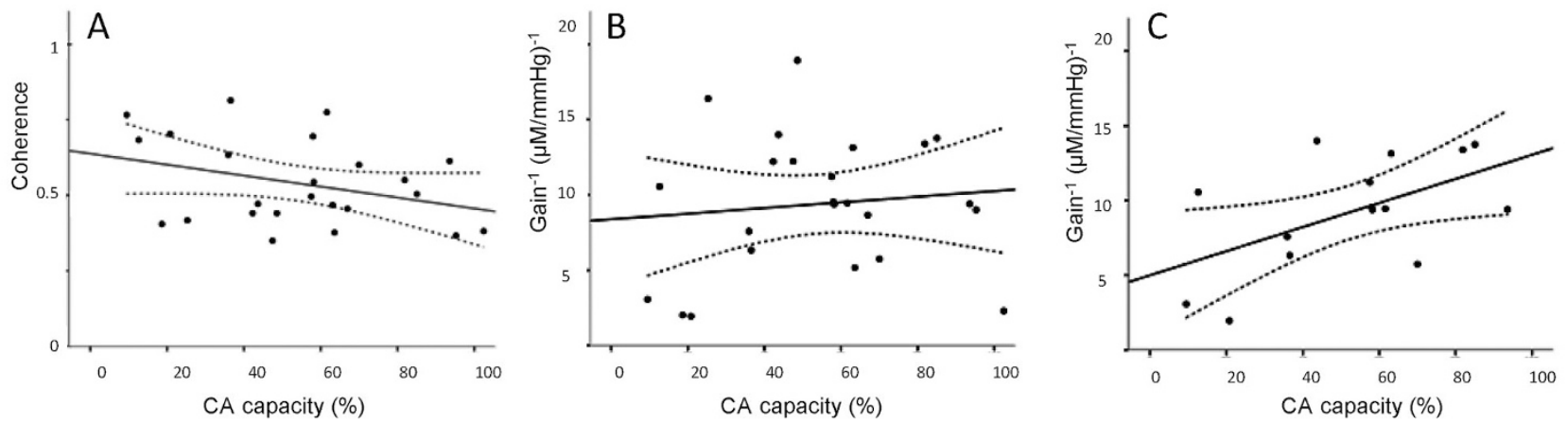

Figure 3. Scatter plots of NIRS-derived measures of CA in the VLF range [coherence $(A)$, gain $(B)$ and gain in the subgroup of measurements with significant coherence $(C)] v s$ LDF-derived CA capacity. Gain was transformed reciprocally to obtain normality. Correlation with coherence $(r=-0.34, n=24, p>0.05)$ and gain $(r=-0.11, n=24, p>0.05)$ was weak. In the subgroup of measurements with significant coherence, however, gain was strongly correlated to CA capacity $(r=-0.55, n=15, p=0.03)$. Dashed lines are $95 \% \mathrm{CI}$. 

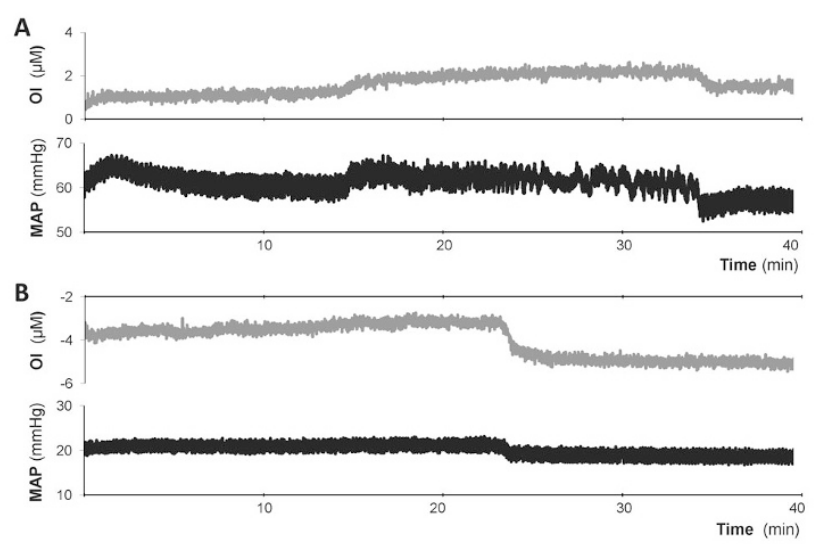

Figure 4. Two recordings of spontaneous ABP-variability (mm Hg; black lines) and corresponding changes in OI ( $\mu \mathrm{M}$; gray lines). Coherence (VLF: $0.003-0.04 \mathrm{~Hz}$ ) was statistically significant during both 40-min measurements ( 0.50 in $A$ vs 0.70 in $B$ ), indicating that CA was not working perfectly. Gain (i.e. the amount of $\mathrm{ABP}$ variation being transferred to the cerebral circulation), however, clearly differed (0.16 in $A$ vs $0.53 \mu \mathrm{M} / \mathrm{mm} \mathrm{Hg}$ in $B)$. CA capacity was $85 \%$ in $A$ and $21 \%$ in $B$.

the cerebral circulation, clearly differed. This difference was reflected in CA capacity changing from 85 to $21 \%$.

\section{DISCUSSION}

This is the first study aiming to validate the use of frequency analysis of cerebral NIRS and ABP to quantify spontaneous autoregulatory vasoreactivity continuously in the neonatal brain. We used a newborn piglet model and demonstrated that this noninvasive method has potential use for measuring $\mathrm{CA}$ in clinical research.

Before we discuss the implications of our findings, several methodological aspects of the study should be considered. First, newborn piglets have anatomical and physiological similarities with the human infant and the developmental stage corresponds to that of a 36- to 38-wk human infant (20). Brain maturation and cerebral hemodynamics in the piglet are similar to that of human infants (21) as is the thickness of the skull. Although the piglet brain is smaller, it is big enough to allow placement of the optodes to ensure that light transverses the brain. Second, the piglets were anesthetized, mechanically ventilated, and usually very stable. This means that the spontaneous variability of ABP often was low leading to a poor signal-to-noise ratio. We used a procedure of weighting that gives periods with high $\mathrm{PSD}$ of $\mathrm{ABP}$, i.e. periods with more variability in $\mathrm{ABP}$, more weight in the final result compared with periods with low PSD of ABP, as this is known to increase the precision of this method (18). Third, to account for the possibility that time under anesthesia may affect the effectiveness of CA, we measured CA capacity both before and after the 40-min period of spontaneous changes in ABP. Fourth, the difference in the way that LDF and NIRS estimate CBF must also be addressed. LDF measures erythrocyte flow velocity in the microvasculature, whereas NIRS measures changes in concentration of oxygenated $\mathrm{Hb}$. Blood flow velocity might only vary over a limited range in these small vessels. Above that range, further increase in CBF will be reached by recruitment of more capillaries for erythrocyte flow. This should be accounted for by the inclusion of the Doppler signal amplitude in the perfusion measure of LDF. Anyway, increased CBF might not be recognized proportionally by LDF and NIRS (22). Fifth, aside from an effectively working CA, an insignificant coherence between spontaneous changes in ABP and cerebral NIRS can basically result from 1) a low variance in $A B P$ and/or 2) a nonlinear relationship between $\mathrm{ABP}$ and cerebral oxygenation (23). Low variance in ABP can lead to a situation where the autoregulatory system is not tested sufficiently and hence the variability in cerebral oxygenation is instead dominated by 1) physiological noise such as variability of arterial content of carbon dioxide, arterial saturation, or cerebral uptake of oxygen or 2) instrumental noise due to limitations of the optics. In our data, however, spontaneous variation in ABP and estimated physiological or instrumental noise did not differ significantly between measurements with and without significant coherence. Sixth, we used OI as a proxy for CBF. This can only be assumed if cerebral oxygen consumption and arterial saturation are kept unchanged (17). In our setting, however, inspired oxygen concentration and mechanical ventilation parameters were held constant to keep arterial saturation at 95 to $98 \%$. Furthermore, we consider cerebral oxygen consumption to be stable during measurements, because our piglets were deeply anesthetized with a constant infusion rate of propofol.

To some surprise, a high coherence between cerebral NIRS and spontaneous ABP changes was only weakly and statistically insignificantly correlated with low CA capacity as measured with LDF during induced ABP changes. However, in the subgroup of measurements with significant coherence (i.e. with a sufficient signal-to-noise ratio), a high gain was associated significantly with low CA capacity in the VLF range. In principle, coherence exceeding the level of significance indicates that $\mathrm{CA}$ is not working perfectly (i.e. that the slope of $\mathrm{CBF}$ against $\mathrm{ABP}$ is not precisely zero), whereas the gain is an estimate of the actual magnitude of this impairment (i.e. the slope of the curve). Consequently, this latter finding was expected.

Measurements in the LF range (i.e. periodic variations occurring over 10-25 s) were only poorly correlated to CA capacity. In line with this finding, Brady et al. (13) in their piglets study found the VLF range sensitive to loss of CA during hypotension, whereas higher frequencies were not. Their finding suggests that the VLF range is useful for studying CA in piglets. The fact that the amount of spontaneous variability in MAP in LF was almost eight times smaller than in the VLF range (i.e. periodic variations occurring over 25-300 s) might partly explain our finding. It is, however, in contrast to an earlier study by our group in preterm infants, where coherence analysis in LF had higher precision and statistically more power than in the VLF range (18). In that study, however, only $9 \%$ were ventilated mechanically. That is, irregular fluctuations in partial pressure of $\mathrm{CO}_{2}$ in arterial blood might have contributed to the lower statistical power in the VLF range.

Our study demonstrates that detection of impaired CA based on significant coherence classifies measurements with minor impairment (high CA capacity and low gain) together with 
measurements with major impairment (low CA capacity and high gain; Fig. 3C). Consequently, association to clinical outcome seems most reasonable for gain and not just coherence.

Clinical studies in preterm infants support the relevance of our findings. In a study by Soul et al. (9), significant coherence alone was frequent but, surprisingly, not associated with IVH. In the subgroup of infants with significant coherence, however, reanalysis demonstrated a significant association between high gain and IVH (10).

Our findings support the concept that CA is a question of quantity and not just quality (24). In other words, CA is not an all-or-none phenomenon but rather a degree of a mechanical response to a change in intravascular pressure. The mechanical response depends on a large number of cellular and molecular mechanisms (1), and it is not surprising that these mechanisms work at varying effectiveness. This concept is supported by a study in preterm infants where xenon clearance was used to measure $\mathrm{CBF}$, finding a CBF-ABP reactivity corresponding to a CA capacity of $75 \%$ in infants who did not develop any ultrasonographic signs of severe IVH (4).

The method is feasible and could be included in the NIRS monitors that are now marketed for clinical use. In patients who already are equipped with an arterial line to monitor $\mathrm{ABP}$, this method requires no additional invasive procedures. The use of spontaneous changes in ABP obviates the need for manipulation of ABP in unstable patients. Unfortunately, as demonstrated in an earlier work by our group (18), its precision is so low that it requires several hours of monitoring to achieve a reliable measurement of coherence. The time needed to estimate gain reliably is not yet known.

In conclusion, our data partly validate frequency analysis for estimation of CA in the newborn brain. In research, a limited precision can be compensated for by larger study populations. For clinical use, the precision, however, seems insufficient.

Acknowledgments. We thank animal health technicians Letty Klarskov and Mette Værum Olesen for their practical help in the experiments. We also thank Senior Research Fellow Terence Leung, Ph.D., from Department of Medical Physics and Bioengineering, University College, London, for programming the software used to carry out the signal analysis.

\section{REFERENCES}

1. Greisen G 2007 Autoregulation of cerebral blood flow. NeoReviews 8:e22-e31
2. Boylan GB, Young K, Panerai RB, Rennie JM, Evans DH 2000 Dynamic cerebral autoregulation in sick newborn infants. Pediatr Res 48:12-17

3. Tyszczuk L, Meek J, Elwell C, Wyatt JS 1998 Cerebral blood flow is independent of mean arterial blood pressure in preterm infants undergoing intensive care. Pediatrics 102:337-341

4. Pryds O, Greisen G, Lou H, Friis-Hansen B 1989 Heterogeneity of cerebral vasoreactivity in preterm infants supported by mechanical ventilation. J Pediatr 115:638-645

5. Lou HC, Lassen NA, Friis-Hansen B 1979 Impaired autoregulation of cerebral blood flow in the distressed newborn infant. J Pediatr 94:118-121

6. Baumbach GL, Heistad DD 1985 Regional, segmental, and temporal heterogeneity of cerebral vascular autoregulation. Ann Biomed Eng 13:303-310

7. Giller CA 1990 The frequency-dependent behavior of cerebral autoregulation. Neurosurgery 27:362-368

8. Elwell CE, Cope M, Delpy DT 1997 An analytical method for determining cerebrovascular transit time using near infrared spectroscopy. Adv Exp Med Biol 428:561-568

9. Soul JS, Hammer PE, Tsuji M, Saul JP, Bassan H, Limperopoulos C, Disalvo DN, Moore M, Akins P, Ringer S, Volpe JJ, Trachtenberg F, du Plessis AJ 2007 Fluctuating pressure-passivity is common in the cerebral circulation of sick premature infants. Pediatr Res 61:467-473

10. O'Leary H, Gregas MC, Limperopoulos C, Zaretskaya I, Bassan H, Soul JS, Di Salvo DN, du Plessis AJ 2009 Elevated cerebral pressure passivity is associated with prematurity-related intracranial hemorrhage. Pediatrics 124:302-309

11. Tsuji M, Saul JP, du Plessis A, Eichenwald E, Sobh J, Crocker R, Volpe JJ 2000 Cerebral intravascular oxygenation correlates with mean arterial pressure in critically ill premature infants. Pediatrics 106:625-632

12. Wong FY, Leung TS, Austin T, Wilkinson M, Meek JH, Wyatt JS, Walker AM 2008 Impaired autoregulation in preterm infants identified by using spatially resolved spectroscopy. Pediatrics 121:e604-e611

13. Brady KM, Lee JK, Kibler KK, Smielewski P, Czosnyka M, Easley RB, Koehler RC, Shaffner DH 2007 Continuous time-domain analysis of cerebrovascular autoregulation using near-infrared spectroscopy. Stroke 38:2818-2825

14. Strebel S, Lam AM, Matta B, Mayberg TS, Aaslid R, Newell DW 1995 Dynamic and static cerebral autoregulation during isoflurane, desflurane, and propofol anesthesia. Anesthesiology 83:66-76

15. Pryds A, Tonnesen J, Pryds O, Knudsen GM, Greisen G 2005 Cerebral pressure autoregulation and vasoreactivity in the newborn rat. Pediatr Res 57:294-298

16. Soul JS, Taylor GA, Wypij D, Duplessis AJ, Volpe JJ 2000 Noninvasive detection of changes in cerebral blood flow by near-infrared spectroscopy in a piglet model of hydrocephalus. Pediatr Res 48:445-449

17. Tsuji M, duPlessis A, Taylor G, Crocker R, Volpe JJ 1998 Near infrared spectroscopy detects cerebral ischemia during hypotension in piglets. Pediatr Res 44:591595

18. Hahn GH, Christensen KB, Leung TS, Greisen G 2010 Precision of coherence analysis to detect cerebral autoregulation by near-infrared spectroscopy in preterm infants. J Biomed Opt 15:037002

19. Tiecks FP, Lam AM, Aaslid R, Newell DW 1995 Comparison of static and dynamic cerebral autoregulation measurements. Stroke 26:1014-1019

20. Chapados I, Cheung PY 2008 Not all models are created equal: animal models to study hypoxic-ischemic encephalopathy of the newborn. Commentary on Gelfand SL et al.: a new model of oxidative stress in rat pups (Neonatology 2008;94:293299). Neonatology 94:300-303

21. Armstead WM 2000 Age-dependent cerebral hemodynamic effects of traumatic brain injury in newborn and juvenile pigs. Microcirculation 7:225-235

22. Klaessens JH, Kolkman RG, Hopman JC, Hondebrink E, Liem KD, Steenbergen W, de Mul FF, Thijssen JM 2003 Monitoring cerebral perfusion using near-infrared spectroscopy and laser Doppler flowmetry. Physiol Meas 24:N35-N40

23. Peng T, Rowley AB, Ainslie PN, Poulin MJ, Payne SJ 2010 Wavelet phase synchronization analysis of cerebral blood flow autoregulation. IEEE Trans Biomed Eng 57:960-968

24. Greisen G 2009 To autoregulate or not to autoregulate-that is no longer the question. Semin Pediatr Neurol 16:207-215 